\title{
Comparison of Kinesio Taping \& Crepe Bandaging In Patients with Post- Operative Pedal Edema: A Randomized Clinical Trial
}

Authors

\author{
Prashant Naik ${ }^{1}$, Pushkar Topkar ${ }^{2}$, Satyashree Vernekar ${ }^{3}$ \\ Lecturer, Dept. of CBR Physiotherapy, KLE Institute of Physiotherapy, Belagavi, Karnataka, India \\ KLE Institute of Physiotherapy, Belagavi, Karnataka, India
}

\begin{abstract}
Background and Objectives: Lymphedema is caused due to the accumulation of interstitial fluid in the tissue spaces due to the inefficiency of the lymphatic system. Kinesiotaping helps in draining of the lymphatic fluid by gently lifting up the skin and allowing the fluid to drain easily into the lymph nodes. Crepe bandaging also helps the draining of lymphatic fluid by creating compressive forces along the limb which helps the fluid to drain in the lymph nodes. The aim of this study is to compare the effectiveness of kinesiotaping and crepe bandaging in patients with post-operative pedal edema.

Materials and Methods: 40 participants with post-operative pedal edema were selected and were allotted in two groups, Group A and Group B each consisting of 20 participants. Group A was given kinesiotaping and Group $B$ was given crepe bandaging and conventional treatment was given to both groups.

Results: The study demonstrated differences in the outcome measure pre and post treatment. Both Group A and Group B showed reduction in pedal edema on both right and left side $(p==0.001)$ which was statistically significant. Comparatively there was a higher reduction in Group A ( $p=0.001)$.

Conclusion: From the present study we can conclude that both kinesiotaping and crepe bandaging give immediate effect by reducing post-operative edema. But kinesio taping is more effective than crepe bandaging in reducing pedal edema in post-operative patients. Hence kinesiotape can be used along with the conventional treatment to relieve edema.

Keywords: kinesiotaping, crepe bandaging, lymphedema, pedal edema, ankle circumference.
\end{abstract}

\section{Introduction}

Lymphedema is the most common complication after surgery ${ }^{1}$. Lymphedema is defined as swelling of a body part due to accumulation of the interstitial tissue fluid ${ }^{2}$.

Primary lymphedema is mainly a congenital disorder caused due to congenital malformation of lymphatic system. Mostly secondary type of lymphedema is seen commonly in clinical practice. Secondary lymphedema is the disorder of lymph flow in lymphatic vessels due to pathological changes in the lymphatic system.
The lymph volume exceeds the lymph transport system indirectly causing accumulation in the extravascular and extracellular fluid in the tissues. The main causes of secondary lymphedema are - Lymph node removal, Trauma to lymphatic system, Irradiation, Inflammation, Compression. ${ }^{2}$ Any type of fracture or trauma is a predisposing factor for lymphedema. Trauma to lower extremity affects bones, muscles and lymphatic system. $^{3}$ 
Kinesio taping was developed by Dr. Kenzo kase a Japanese chiropractor. It is an elastic tape that can augment the current treatments such as massage manual lymphatic drainage medical compressive garments positional consideration \&/or exercises. ${ }^{4,5}$

The concept of kinesio taping was that the proposed therapy should affect the persons not only during therapist's visit but also throughout the day hence he developed a type of support in the form of kinesio tape ${ }^{4}$.

This tape is proven to be effective as the tape application cause delicate lifting of the skin which increases the space between corium and fascia. ${ }^{6}$ This lifting effect helps in forming convolutions in the skin which increases the interstitial space causing decrease in inflammation of the affected areas. ${ }^{7}$

Compression therapy with the help of elastic or non-elastic compression bandages are used as they provide low compressive forces to the extremity at rest. Crepe bandages are used as a form of compression therapy to help in draining the lymphatic fluid into the collateral channels. ${ }^{2}$ It facilitates a mild increase in tissue pressure assisting lymph vessels to empty which further prevents refilling of the interstitium improving the efficiency of the muscle pump during activity and providing localized pressure indicated to soften fibrotic tissue. ., $^{8}$

To evaluate and clinically assess pedal edema there are different methods of assessment. The methods to assess pedal edemain ankle circumference the circumference is measured at single location in which each ankle is marked with a semi-permanent marker at approximately $7 \mathrm{~cm}$ above the medial malleolus and ankle circumference is measured in centimeters. Ankle circumference shows higher inter examiner agreement and excellent reliability. ${ }^{9}$

Treatment of lymphedema involves the combination of medical management and physiotherapy management combined with selfmanagement by the patient. ${ }^{10}$ Physiotherapy management of lymphedema includes pneumatic compression treatment, exercises, massage. ${ }^{11}$

\section{Materials and Methods \\ Institutional review board approval}

This study got approval from institutional review board and was conducted in conformity with the ethical and human principles of research.

\section{Participants}

40 participants were included in the study from KLE Prabhakar Kore Hospital and MRC from October 2015 to February 2016. The inclusion criteria were: 1 . Patients with post-operative pedal edema. The exclusion criteria were: 1 . Associated neurological symptoms. 2. Fragileskin, 3.Deep vein thrombosis, 4. Varicose veins, 5. Open wounds, 6. Coronary artery bypass grafting, 7.Renal surgeries, 8. Hypertension

\section{Procedure}

Participants who reported to KLES Dr. Prabhakar Kore Hospital and MRC, Belgaum with postoperative pedal edema were screened for their eligibility depending on inclusion and exclusion criteria. After finding their suitability, they were requested to participate in the study and were given a written informed consent. After obtaining their consent, demographic data including age, gender, nature of symptoms and occupation, other specific data such as initial ankle circumference was measured and then were randomly allocated to one of the two study groups viz A and B

Group A: Kinesiotaping + conventional treatment of elevation and ATMs.

Group B: Crepe bandaging + conventional treatment of elevation and ATMs.

\section{Results}

Statistical Analysis: Statistical analysis for the present study was done manually as well as using statistical package of social science version 16 in order to verify the results obtained. Statistical measures such as mean, standard deviation and tests of significance such as Chi-square test, paired and unpaired ' $t$ ' test, were utilized for all the scores in the participants. Participants included in the study were in the age 
group of 18-70 years. Mean age of group A was $40.17 \pm 15.61$ years and mean age of group B was $43 \pm 14.34$ years.

40 participants were included in the clinical trial of which 20 participants present in group A included 11 males and 9 females and 20 participants of group B included 11 males and 9 females

Table 1: Comparison of ankle circumference on right side between group A and group B

\begin{tabular}{|c|c|c|c|c|c|c|c|c|}
\hline \multirow[t]{2}{*}{ Group } & \multicolumn{2}{|c|}{ Pre-treatment } & \multicolumn{2}{|c|}{$\begin{array}{c}\text { Post - } \\
\text { treatment }\end{array}$} & \multicolumn{2}{|c|}{ Difference $\downarrow$} & \multirow[t]{2}{*}{$\mathrm{T}$} & \multirow[t]{2}{*}{$\mathrm{p}$} \\
\hline & Mean & SD & Mean & SD & Mean & SD & & \\
\hline A & 21.62 & \pm 1.49 & 20.84 & \pm 1.50 & 0.73 & \pm 0.26 & 9.49 & $<0.001+$ \\
\hline $\mathrm{B}$ & 21.27 & \pm 2.29 & 20.82 & \pm 2.23 & 0.45 & \pm 0.18 & 10.33 & $<0.001+$ \\
\hline $\mathrm{T}$ & \multicolumn{2}{|c|}{0.525} & \multicolumn{2}{|c|}{0.024} & \multicolumn{2}{|c|}{3.561} & & \\
\hline $\mathrm{P}$ & \multicolumn{2}{|c|}{0.599} & \multicolumn{2}{|c|}{0.981} & \multicolumn{2}{|c|}{$<0.001+$} & & \\
\hline
\end{tabular}

Table 2: Comparison of ankle circumference on left side between group A and group B

\begin{tabular}{|c|c|c|c|c|c|c|c|c|}
\hline \multirow[t]{2}{*}{ Group } & \multicolumn{2}{|c|}{ Pre-treatment } & \multicolumn{2}{|c|}{$\begin{array}{l}\text { Post - } \\
\text { treatment }\end{array}$} & \multicolumn{2}{|c|}{ Difference $\downarrow$} & \multirow[t]{2}{*}{$\mathrm{T}$} & \multirow[t]{2}{*}{$\mathrm{p}$} \\
\hline & Mean & SD & Mean & SD & Mean & SD & & \\
\hline A & 21.62 & \pm 1.49 & 20.84 & \pm 1.50 & 0.73 & \pm 0.26 & 9.49 & $<0.001^{+}$ \\
\hline $\mathrm{B}$ & 21.27 & \pm 2.29 & 20.82 & \pm 2.23 & 0.45 & \pm 0.18 & 10.33 & $<0.001^{+}$ \\
\hline $\mathrm{T}$ & \multicolumn{2}{|c|}{0.525} & \multicolumn{2}{|c|}{0.024} & \multicolumn{2}{|c|}{3.561} & & \\
\hline $\mathrm{p}$ & \multicolumn{2}{|c|}{0.599} & \multicolumn{2}{|c|}{0.981} & \multicolumn{2}{|c|}{$<0.001^{+}$} & & \\
\hline
\end{tabular}

Graph 1: Ankle circumference within and between Group A and Group B of right side

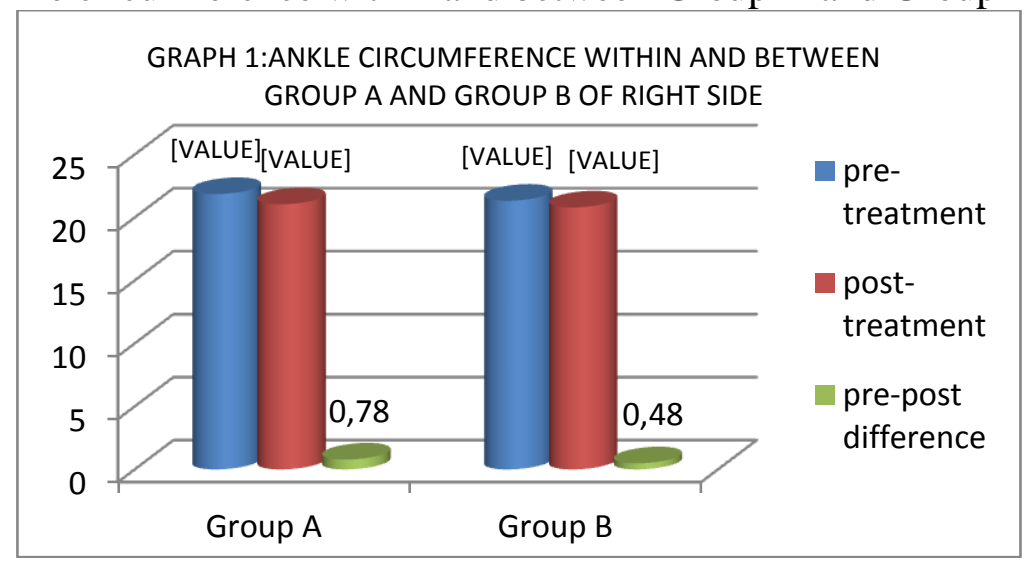

Graph 2: Ankle circumference within and between Group A and group B of left side

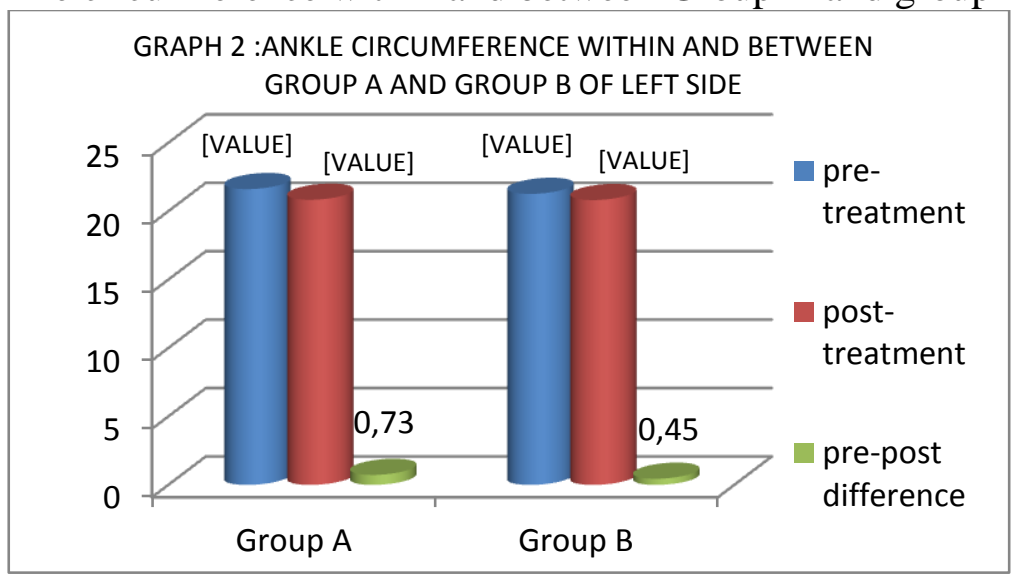




\section{Discussion}

The present study was proposed to compare the effectiveness of kinesio taping \& crepe bandaging in patients with post-operative pedal edema: A randomized clinical trial".

Lymphedema is defined as swelling of a body part due to accumulation of the interstitial tissue fluid.

Crepe bandages are used as a form of compression therapy to help in draining the lymphatic fluid into the collateral channels. ${ }^{2}$ lymph edema bandaging is a highly specialized form of bandaging which utilizes multiple layers of short stretched bandages for lymph edematous body segments. ${ }^{6}$

Till date the conventional treatment to relive pedal edema was elevation and ankle toe movements. Kisner C, Colby LA. in their study used elevation and ankle toe movements as a exercising tool in treatment of edema and concluded that elevation and ankle toe movements are helpful in reduction of edema,. Keeping this in mind in the present study elevation for $30 \mathrm{~min}$ and 10 repetition of ankle toe movements was given as a conventional treatment ${ }^{2}$.

Ankle circumference is most reliable and valid tool to measure pedal edema. ${ }^{9}$ So in the present study ankle circumference was used to measure the pedal edema.

In this study crepe bandage and kinesio tape was used to relive edema. In earlier studies compression stockings was used in hospital nurses to reduce edemawhereas fiberglass taping therapy was useful in reducing pain. Hence it was concluded that these techniques should be used frequently in clinical practice. ${ }^{11}$

As we know in edema the skin nourishment is hampered due to immobility and the edematous skin is fragile on which any normal taping cannot be used as it may damage the edematous skin. Kinesio taping on edema is safe and can be tolerated by the tissue and the patient, as one study done by Martins $\mathrm{J}$ de $\mathrm{C}$, et al. about the safety and tolerability of kinesio taping in patients
Kinesio taping is an elastic tape which provides a directional pull that guides the lymphatic fluid in the desired direction of drainage. This is important in routing and rerouting the lymphatic fluid in lymphatic vessels to reduce swelling. Tape can continue facilitating lymphatic flow for the next 3-4 days during the wearing of the tape after the therapy session. ${ }^{7}$

with arm lymphedema. Found that kinesio taping technique was safer and tolerable in patients with lymphedema. ${ }^{14}$ so in present study kinesio taping was very effective and safe in treatment of pedal edema.

Kinesio tape was used to relive post-operative pedaledema, in similarly study done by Lipińska A, et al. observed and concluded that the lymphatic applications of kinesiotaping help in reducing lymph edema. ${ }^{4}$

It is mostly seen that in cases of pedal edema crepe bandage is one of the fevourate tool for reduction of edema. The application of crepe bandage showed reduction in pedal edema. Similarly a study by Alizadeh-Ghavidel A, et al. they concluded that the compression stocking helps to relive edema and wound complications after the surgery. ${ }^{12}$

As we know kinesio taping and crepe bandaging reduces edema. A study done on combined effects of compression bandage along with kinesio taping on lymphedema, showed excellent reduction in volumeand edema. ${ }^{16}$ simillarly in present study comparison was done between kinesio tape and crepe bandage to find its effectiveness in reduction of edema.

Application of crepe bandaging is one of the safest method in reducing edema. Bowering, Keith C. MD, FRCP to check the effectiveness of layered compression bandages concluded that layered compression bandages was an effective and safe treatment for patients and helped to reduce edema. $^{13}$

Application of kinesio tape reduces lymph edema which leads to increase mobility. Maha.A.Hassan, Samah Ismail. Compared the effect of kinesio 
taping and compression garment on post mastectomy lymphedema which concluded that both therapies showed equal effect on the reduction of lymphedema, hence improving the quality of life, and also shows reduction in pain. ${ }^{15}$

As per the review of literature no study has compared the effects of kinesio taping and crepe bandaging to relive post-operative pedal edema. In the present study the comparison of kinesio taping and crepe bandaging was done to check its effectiveness. When the comparison was done in two groups it showed that there was more reduction in pedal edema in participant's subjected to kinesio taping with conventional treatment as compared to the participants who received crepe bandaging with conventional treatment which was measured by the ankle circumference in centimeters. This was due to the lifting effect of the kinesiotaping which helped in more lymphatic drainage as compared to crepe bandaging which helped in compression and return of the lymphatic fluid.

Limitations

\section{Conclusion}

From the present study we can conclude that both kinesiotaping and crepe bandaging give immediate effect by reducing post-operative edema. But kinesio taping is more effective than crepe bandaging in reducing pedal edema in postoperative patients. Hence kinesiotape can be used along with the conventional treatment to relieve edema.

Future Scope: The present study can be conducted on a large sample size.

Specific type of post-operative conditions.

The period of intervention can be longer to know the long term effects with a further follow up.

Conflicts of interest: None

\section{References}

1. Edem - Wikipedia, the free encyclopedia [Internet]. [cited 2015 Oct 11]. Available from: https://en.wikipedia.org/wiki/Edem
2. Kisner C, Colby LA. Therapeutic Exercise: Foundations and Techniques. F.A. Davis; 2007. ISBN 978-0-80363897-6 page no 834-838 .

3. Szczesny G, Olszewski WL. The Pathomechanism of Posttraumatic Edema of the Lower Limbs: II???Changes in the Lymphatic System: The Journal of Trauma: Injury, Infection, and Critical Care. 2003 Aug;55(2):350-4.

4. Lipińska A, Śliwiński Z, Kiebzak W, Senderek T, Kirenko J. The influence of Kinesiotaping applications on lymphoedema of an upper limb in woman after mastectomy. Fizjo Pol. 2007;7(3):258-69.

5. Tsai H-J, Hung H-C, Yang J-L, Huang C-S, Tsauo J-Y. Could Kinesio tape replace the bandage in decongestive lymphatic therapy for breast-cancerrelated lymphedema? A pilot study. Support Care Cancer. 2009 Feb 8;17(11):1353-60.

6. Chou Y-H, Li S-H, Liao S-F, Tang HW. Case Report: Manual Lymphatic Drainage and Kinesio Taping in the Secondary Malignant Breast CancerRelated Lymphedema in an Arm With Arteriovenous (A-V) Fistula for Hemodialysis. American Journal of Hospice and Palliative Medicine. 2013 Aug 1;30(5):503-6

7. Stockheimer KR. Kinesio taping and lymphoedema. Advance Healing. 2006;3:22-3

8. Thomas S. Compression bandaging in the treatment of venous leg ulcers. World Wide Wounds. First publication. 1997.

9. Kazmi SSH, Stranden E, Kroese AJ, Slagsvold C-E, Diep LM, Stromsoe K, et al. Edema in the Lower Limb of Patients Operated on for Proximal Femoral Fractures: The Journal of 
Trauma: Injury, Infection, and Critical Care. 2007 Mar;62(3):701-7.

10. Cash JE. Cash's Textbook of Chest, Heart, and Vascular Disorders for Physiotherapists. Lippincott; 1979.ISBN 978-0-397-58250-1 662 p- 683p.

11. Kang S-A, Chon M-Y. Comparative Effectiveness of Taping Therapy Versus Compression Stocking on Edema, Pain, and Fatigue in the Lower Extremities of Hospital Nurses. Indian Journal of Science and Technology. 2015;8(S8):15-21.

12. Alizadeh-Ghavidel A, Ramezannejad P, Mirmesdagh Y, Sadeghpour-Tabaei A. Prevention of Edema After Coronary Artery Bypass Graft Surgery by Compression Stockings. Research in Cardiovascular Medicine. 2014 May;3(2).

13. Bowering KC. Use of Layered Compression Bandages in Diabetic Patients: Experience in Patients with Lower Leg Ulceration, Peripheral Edema, and Features of Venous and Arterial Disease. Advances in Skin \& Wound Care. 1998 May 1;11(3):12935.

14. Martins J de C, Aguiar SS, Fabro EAN, Costa RM, Lemos TV, Sá VGG de, et al. Safety and tolerability of Kinesio® Taping in patients with arm lymphedema: medical device clinical study. Support Care Cancer. 2015 Aug 14;1-6.

15. HASSAN MA, ISMAIL SM. Kinesio Tape Versus Compression Garment on Post Mastectomy Lymphedema.

16. Key mr. Can coban 2 compression system in combination with kinesio taping make a difference in oedema reduction. 